\title{
Effect of Saline Irrigation Water and Organic Waste Quality on Some Growth and Yield Parameters of Barley
}

\author{
Sabah Lateef Asi ${ }^{\mathrm{a}} \quad$ Mohammed Tarkhan Abo - Almeekh ${ }^{\mathrm{b}}$ Hammed Kadhum Abd - Al Ameer \\ ${ }^{a, b, c}$ Department of Soil and Water Technology, Technical College Musayab, Al-Furat University Middle \\ Tech, Soil and Water Technology, Technical College Musayb, Al-Furat Middle Technical University, \\ Babil, Iraq. \\ sabah_alshammary@tem.edu.iq
}

Submission date:- 7/3/2018 Acceptance date:- 4/4/2018 Publication date:- 14/10/2018

Keywords: Organic waste, Drainage water, Barle, Al-Warkaa.

\begin{abstract}
Field experiment was conducted in Al-Yosfia region at 2016-2017 in loamy soil to investigate the effect of three level of saline water (River water, drainage water $4.3 \mathrm{dS} . \mathrm{m}^{-1}$, and drainage water 7.2 $\mathrm{dS} . \mathrm{m}^{-1}$ ) and three types of analytical organic fertilizer (Poultry waste ,cow waste, and Ground corn cobs) with 12 ton. $\mathrm{h}^{-1}$ and their interaction on some growth and yield parameters of Barley Al-Warkaa Var., the experiment at RCBD with three replicates using LSD test at 5\% to compare between parameter means.
\end{abstract}

The results showed that when using saline irrigation water, the values of growth and yield parameter decrease while organic waste reduce the negative effect of saline irrigation water, the poultry waste gave significant increase of parameter values followed by cow waste then ground corn cobs. The interaction between (Poultry waste and river water ) gave higher means to plant height, vegetative dry matter, leaf area, chlorophyll content, No. of spike. $\mathrm{m}^{-2}$, No. of seeds per spike, weight of 1000 seeds, total seeds yield, biological yield, and harvesting index. The value was $98.4 \mathrm{~cm}, 17.4 \mathrm{~g}, 27.4 \mathrm{~cm}^{2}$, $51.2 \mathrm{spad}, 332.6$ spike. ${ }^{2}$, 38.2 seed per spike, $38.2 \mathrm{~g}, 4.853$ ton.h $\mathrm{h}^{-1}, 15.423$ ton.h $^{-1}$, and $31.47 \%$ respectively.

\section{تأثير ملوحة ماء الري ونوعية المخلفات العضوية في بعض مؤشرات نمو وحاصل الثعير

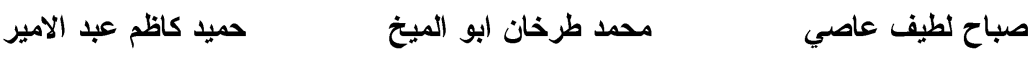




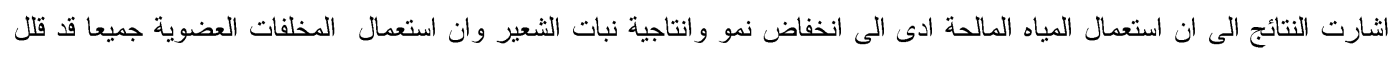

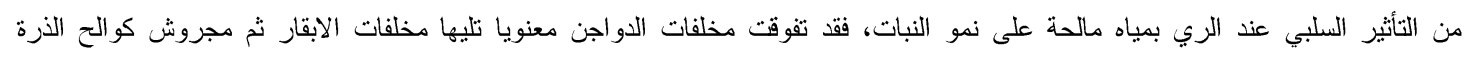

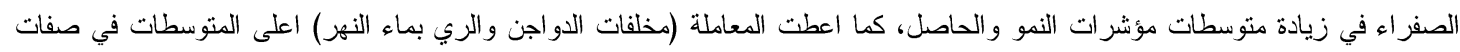

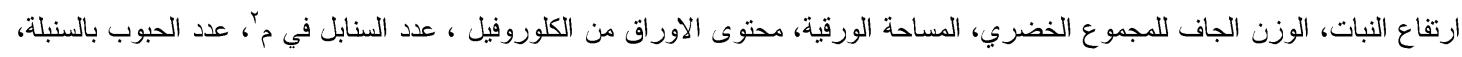

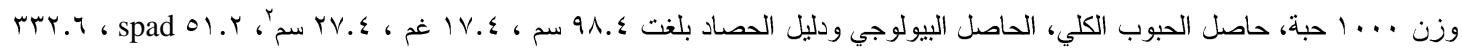

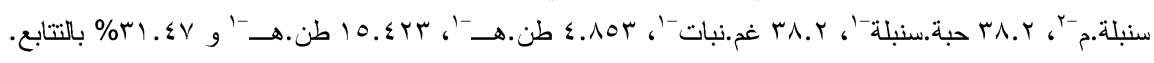
الكلمات الالة: مخلفات عضوية، مياه بزل، شعير، الوركاء.

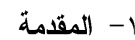

يعد محصول الثعير .Hordeum vulgare L بأصنافه المختلفة من المحاصيل النجيلية وهو رابع المحاصيل الاستر اتيجية في العالم بعد

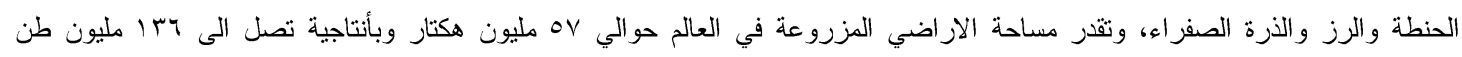

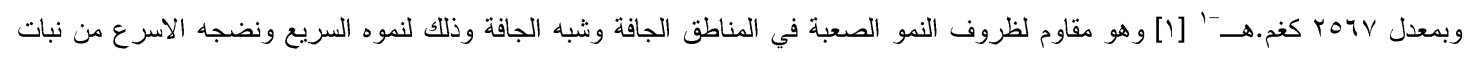

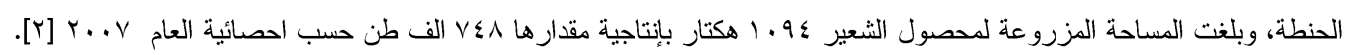

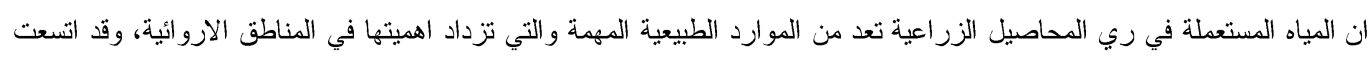

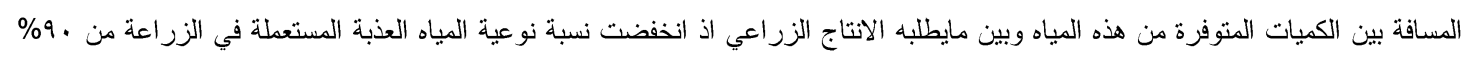

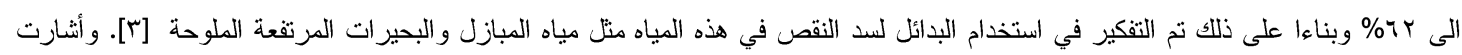

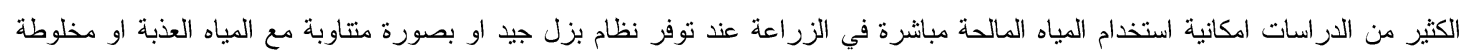

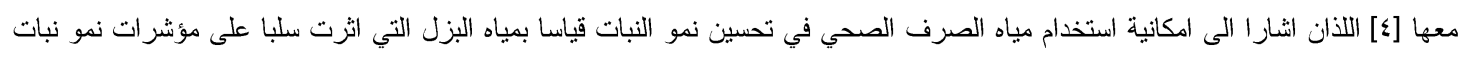

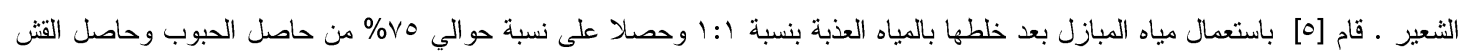
لنبات الثعير قياسا عند استعمال المياه العذبة لوحدها.

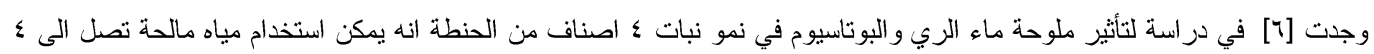

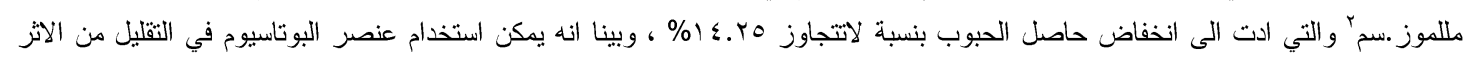

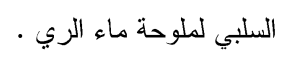

ان المادة العضوية هي مخلفات النباتات والحيو انات وما تحويه التربة من كائنات حية وعند توفر الظروف الملائمة من رطوبة وحرارة

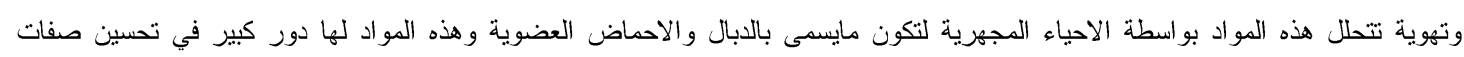

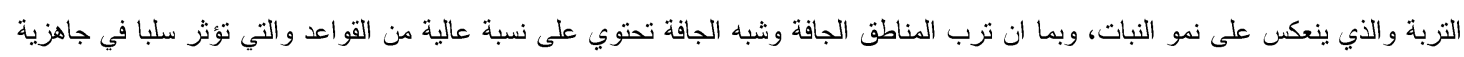

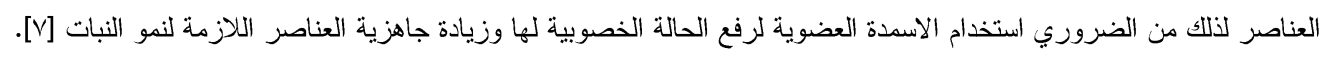
ان استخدام الاسمدة المعدنية بصورة مستمرة ادى الى حدوث مشاكل في صفات التربة لذا اصبح الزاما في البحث عن بدائل عن التسميد

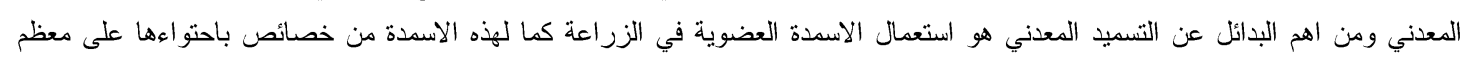

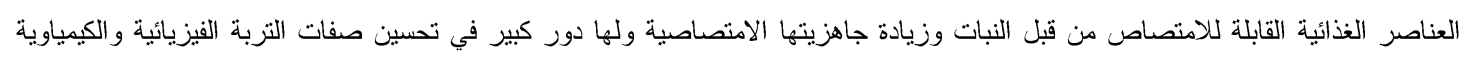

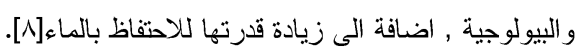

وجد [9] ان استعمال مخلفات القصب البري المطحون كمادة عضوية الى التربة وبمستويات مختلفة ادى الى تقليل ملوحة التربة وزيادة

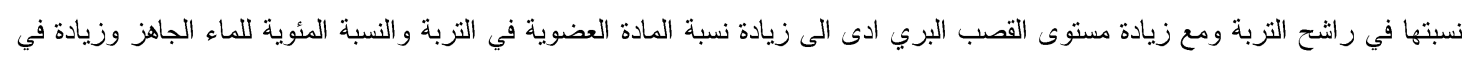

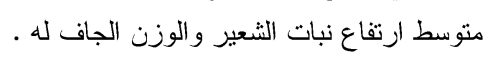

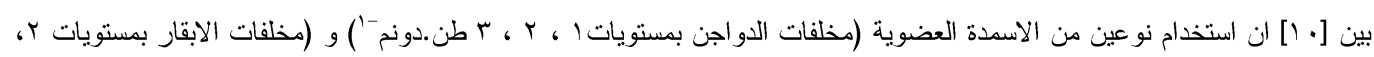

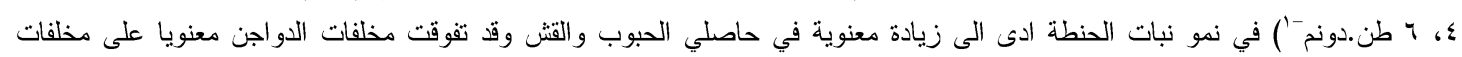
الابقار.

تهدف الدراسة الى استعمال انواع مختلفة من الاسمدة العضوية (المخلفات الحيو انية والكوالح ) للنقليل من الاثر السلبي للمياه المالحة المستعملة في ري محصول الثعير. 


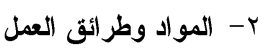

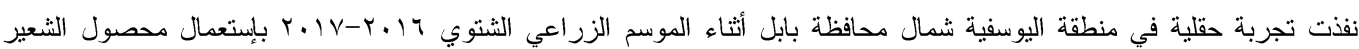

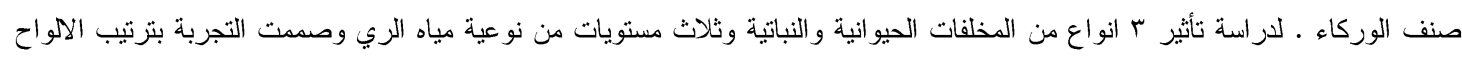

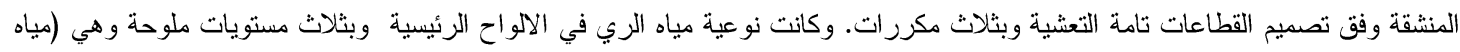

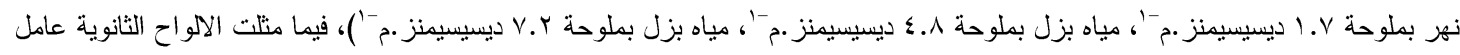

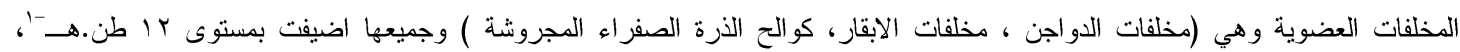

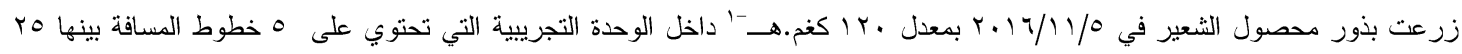

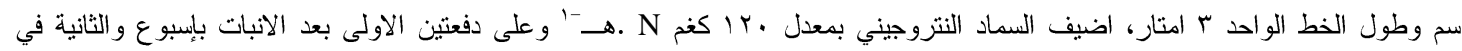

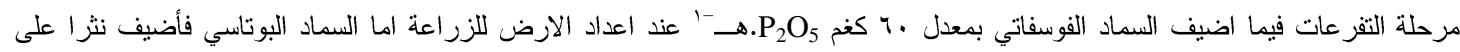

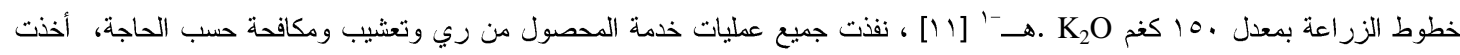

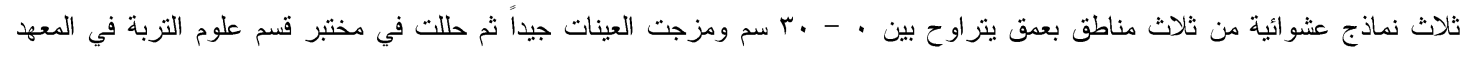

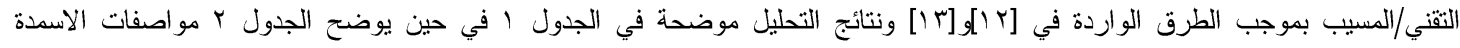

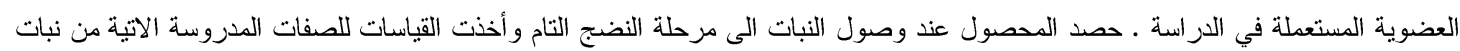

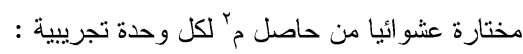
1-ارتفاع النبات (سم) وقيس كمتوسط لعشرة نباتات ومن اتصال النبات في سطح التزبة الى نهاية السنبلة عدا السفا .

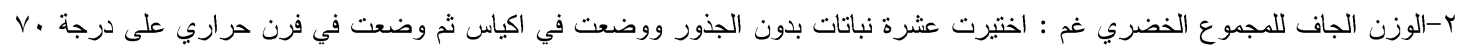

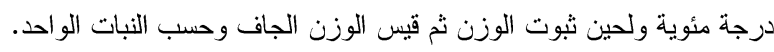
ب-مساحة ورقة العلم سم : حسبت كمتوسط لعشرة اوراق علم في الوحدة التجريية وبحسب المعادلة الاتية: مساحة ورقة العلم سم = طول ورقة العلم * العرض من المنتصف * 90. ع-محتوى الاور اق من الكلوروفيل spad : قدر بجهاز Chlorophyll meter نوع SPAD موقعيا وعلى النبات مباشرة وذلك بأخذ معدل ثلاث قراءات لكل ورقة .

ه-عدد السنابل في المتر المربع : حسبت من مجموع السنابل للنباتات المحصودة في مساحة مُّ الواحد. ؟-عدد الحبوب في السنبلة :حسبت كمتوسط لعدد الحبوب في • ( سنابل اخذت عشو ائيا من النباتات المحصودة ضمن مُّم لا-وزن . . ا حبة غم : حسبت بميزان حساس وكمتوسط لثلاثة نماذج اخذت من السنابل المحصودة . ^-الحاصل الكلي طن.هـ-' : حسبت عدد السنابل وعدد الحبوب ووزن . .0 حبة في الـ مَّ ثم حول الناتج الى طن.هـ-'. 9-الحاصل البايولوجي طن.هـ -' : ويشمل حاصل القش و الحبوب وحسب من وزن النباتات المأخوذة في مُ ثخ حول الناتج الى طن.هــ-. • ا-سليل الحصاد \% : حسب من حاصل قسمة الحاصل الكلي مقسوما على الحاصل البايولوجي مضروبا في . . . . حللت النتائج احصائيا حسب الطرق الواردة في [0 10] واختبرت المتوسطات حسب اختبار اقل فرق معنوي وبمستوى ه.. . . وأستخدم برنامج [ 1 1 ] في تتفيذ التحليل. 
جدول (1) مواصفات التربة المستعملة في التجربة

\begin{tabular}{|c|c|c|}
\hline القيمة & وحدة القياس & الصفة \\
\hline r.А & ديسي سيمنز •م -1 & التوصيل الكهربائي \\
\hline$V .7$ & --- & تفاعل التربة \\
\hline$\wedge . \varepsilon$ & غم ـ كغم ־'تزبة & المادة العضوية \\
\hline 19.8 & سنتي مول . كغر' & السعة التبادلية \\
\hline$r \ldots r$ & غم ـ كغم -1 تربة & كاربونات الكالسيوم \\
\hline rr.t & \multirow{3}{*}{ ملغم.كغم -' تربة } & النتروجين الجاهز \\
\hline $1 Y .0$ & & الفسفور الجاهز \\
\hline 1.9 .8 & & البو تاسيوم الجاهز \\
\hline 1.4 & ميكاغر ام · م- مي & الكثافة الظاهرية \\
\hline MIV.o & \multirow{3}{*}{ غم • كغم -'تزبة } & 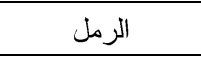 \\
\hline$\sum 7 V . Y$ & & الغرين \\
\hline Ylo.r & & الطين \\
\hline مزيجة & & النسجة \\
\hline
\end{tabular}

جدول (ץ) بعض مواصفات المخلفات العضوية المستعملة في الار اسة

\begin{tabular}{|c|c|c|c|c|c|c|c|}
\hline $\mathbf{C} / \mathbf{N}$ & الكاربون العضوي & البوتاسيوم \% & الفسفور & \% النتروجين \% & pH & Ec 1:1 & نوع المخلفات \\
\hline Ir.ro & YA.qY & Y.IY & 1.19 & T.rE & 7.7 & 7.1 & الدو اجن \\
\hline 15.99 & 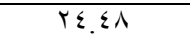 & r.Y & 1.14 & $1 . \wedge \mathrm{V}$ & 7.9 & $0 . \varepsilon$ & الابقار \\
\hline$r .00$ & $\varepsilon \varepsilon .7$ & 9.07 & $\varepsilon . \wedge \mu$ & r.IV & 7.9 & $\varepsilon . \wedge$ & الكو الح \\
\hline
\end{tabular}

r- - ب النتائج والمناقشة

1. ا ارتفاع النبات (سم) والوزن الجاف للنبات (غم)

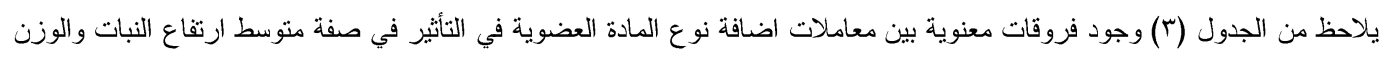

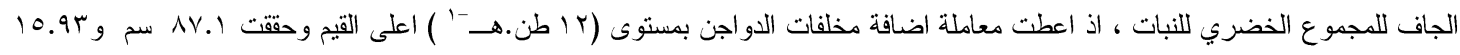

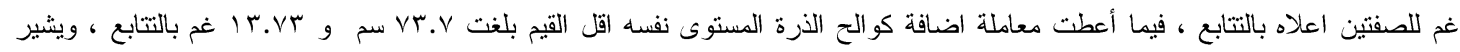

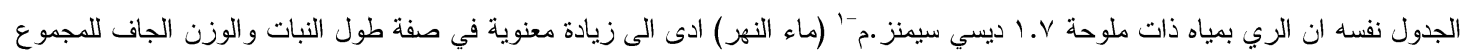

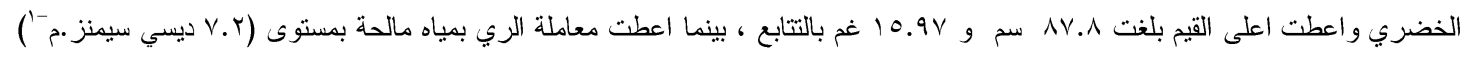

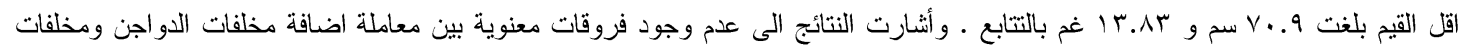

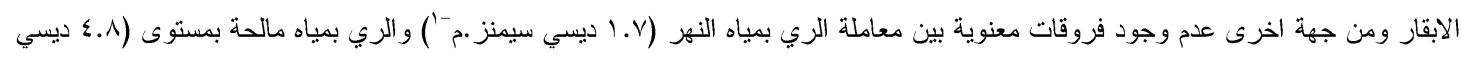

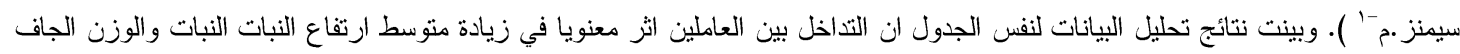

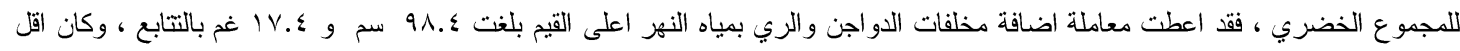

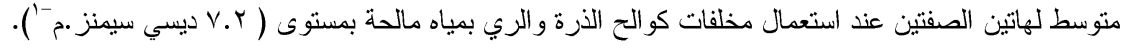


جدول (r) تأثير نوع المخلفات وملوحة ماء الري وتداخلهما في متوسط ارتفاع النبات والوزن الجاف للنبات

\begin{tabular}{|c|c|c|c|c|c|c|c|c|}
\hline \multicolumn{4}{|c|}{ الوزن الجاف للنبات غم } & \multicolumn{4}{|c|}{ ارتفاع النبات سم } & \multirow{3}{*}{ نوع المخلفات } \\
\hline \multicolumn{4}{|c|}{ ملوحة ماء الزي } & \multicolumn{4}{|c|}{ dS.m-1 ملوحة ماء الري } & \\
\hline المتوسط & V.Y & $\varepsilon . r$ & $1 . r \wedge$ & المتوسط & V.r & ๕.r & $1 . r \wedge$ & \\
\hline 10.94 & $1 \varepsilon . r$ & 17.1 & IV. & $\wedge \vee . ı$ & VY.V & $9 . . r$ & $9 \wedge . \varepsilon$ & 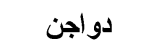 \\
\hline $10 . r V$ & $1 \varepsilon . r$ & $10 . Y$ & $17 . \%$ & Al.V & V1.1 & 10.r & $\wedge \wedge .7$ & ابقار \\
\hline \multirow[t]{2}{*}{ ir.vr } & $1 r .9$ & $1 \varepsilon .1$ & $1 \varepsilon . r$ & Vr.V & $1 \wedge .9$ & $\vee \bullet . \Lambda$ & V५.ร & 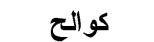 \\
\hline & Ir.Ar & $10.1 \%$ & $10.9 \mathrm{~V}$ & & $v \cdot .9$ & $\wedge r . \wedge$ & $\wedge \vee . \wedge$ & المتوسط \\
\hline \multicolumn{3}{|c|}{ تداخل:9.1.1 } & مخلقات:9 . & \multicolumn{3}{|c|}{ ماء الري:؛ \&.V تداخل:r.r. } & مخلفات: ؛ ؛ 1 & LSD .05 \\
\hline
\end{tabular}

r.r معدل مساحة ورقة العلم (سمَّ) ومحتوى الاوراق من الكلوروفيل

يلاحظ من الجدول (ع) وجود فروقات معنوية بين معاملات اضافة نوع المادة العضوية في التأثير في صفة منوسط المساحة الورقية

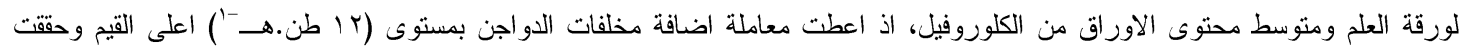

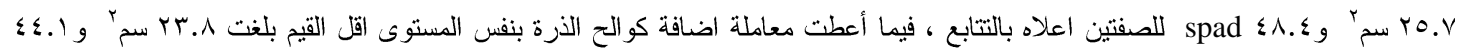

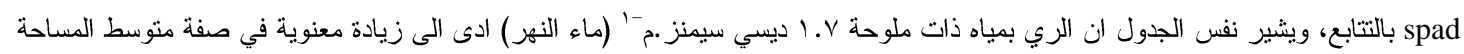

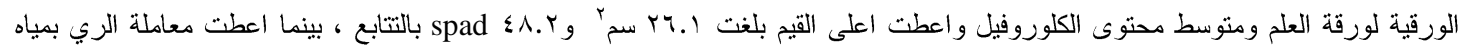

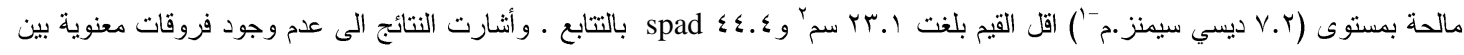

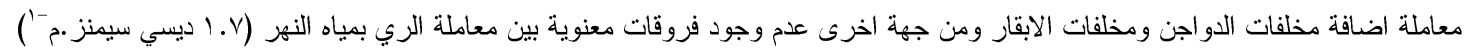

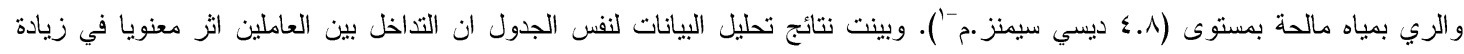

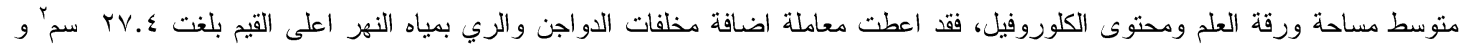

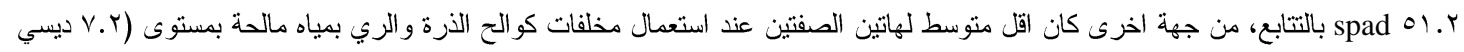

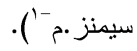

جدول (؛) تأثير نوع المخلفات وملوحة ماء الري وتداخلهما في متوسط المساحة الورقية ومحتوى الكلوروفيل

\begin{tabular}{|c|c|c|c|c|c|c|c|c|}
\hline \multicolumn{4}{|c|}{ Sحتوى الكلوروفيل Spad } & \multicolumn{4}{|c|}{ المساحة الورقية لورقة العلم سمَ } & \multirow{3}{*}{ المخلفات } \\
\hline \multicolumn{4}{|c|}{ ملوحة ماء الري } & \multicolumn{4}{|c|}{ ملوحة ماء الري } & \\
\hline المتوسط & V.r & $\varepsilon . r$ & $1 . r \wedge$ & المتوسط & V.r & $\varepsilon . r$ & $1 . r \wedge$ & \\
\hline$\{\wedge . \wedge$ & $\{4 . \wedge$ & $\varepsilon \wedge . r$ & $01 . r$ & ro.v & rr.o & 4.1 & YV.s & دواجن \\
\hline$\varepsilon v .1$ & $\varepsilon \varepsilon .9$ & $\varepsilon V .7$ & $\{\wedge . \wedge$ & ro.. & rr. & ro.1 & YY.r & ابقار \\
\hline \multirow[t]{2}{*}{$\varepsilon \varepsilon .1$} & $\{1.7$ & $\leqslant 0.9$ & $\varepsilon \varepsilon . V$ & rr.A & rY.A & $r \varepsilon .1$ & $r \varepsilon .7$ & كوالح \\
\hline & $\{\varepsilon . \varepsilon$ & $\varepsilon V . r$ & $\{\Lambda . Y$ & & rr.l & ro.r & Y..1 & المتوسط \\
\hline \multicolumn{2}{|c|}{ تذاخل:؛ r.ب } & كاء الرج & مخلقات:1. 1 1. & \multicolumn{2}{|c|}{ 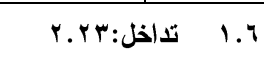 } & ماء & مخلقات: ؛ & LSD .05 \\
\hline
\end{tabular}

\section{r.r متوسط عدد السنابل .مَّ وعدد الحبوب في السنبلة}

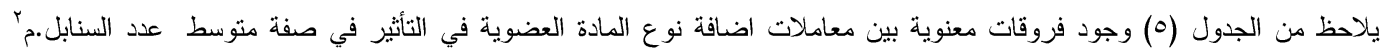

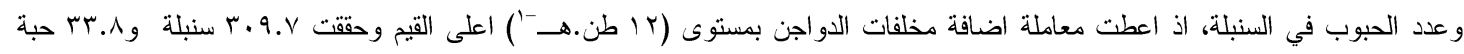

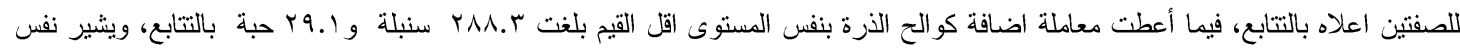

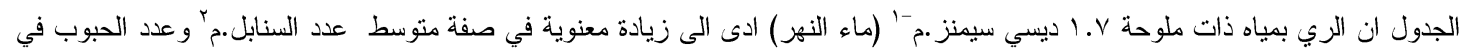

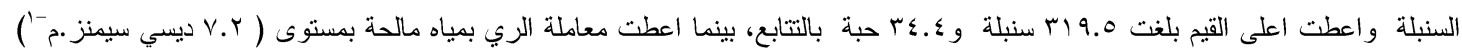

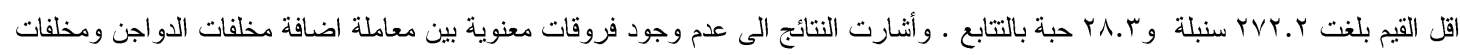

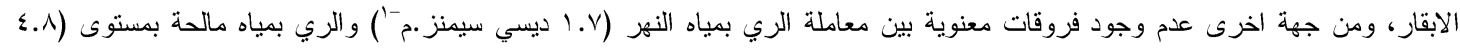

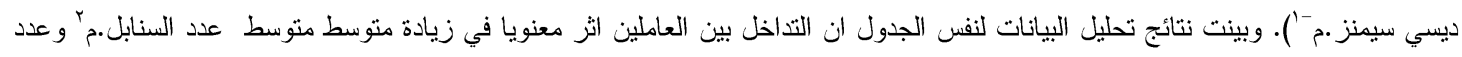




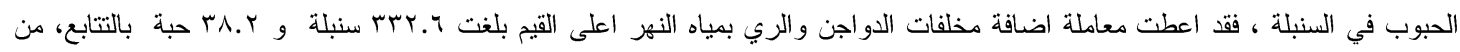

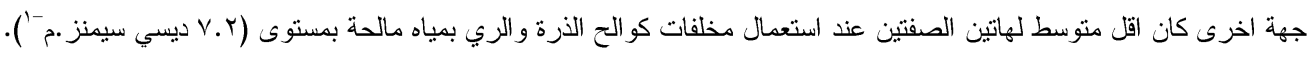

جدول(•) تأثير نوع المخلفات وملوحة ماء الري وتداخلهما في متوسط عدد السنابل مُ وعدد الحبوب.سنبلة-1

\begin{tabular}{|c|c|c|c|c|c|c|c|c|}
\hline \multicolumn{4}{|c|}{ عدد الحبوب.سنبلة- } & \multicolumn{4}{|c|}{ عدد السنابل. مُ } & \multirow{3}{*}{ نوع المخلفات } \\
\hline \multicolumn{4}{|c|}{ dS.m-1 ملوحة ماء الري } & \multicolumn{4}{|c|}{ dS.m-1 ملوحة ماء الزي } & \\
\hline المتوسط & V.r & $\varepsilon . r$ & $1 . r \wedge$ & المتوسط & V.r & $\varepsilon . r$ & $1 . r \Lambda$ & \\
\hline 的.A & ra.1 & rr.o & rA.r & $r .9 . v$ & rVq.r & TIV.\& & Tru.t & دواجن \\
\hline rr.e & YV.q & rr.v & $r \varepsilon . \theta$ & r.Y.r & $r V \cdot . A$ & $r . . r$ & rus.o & ابقار \\
\hline \multirow[t]{2}{*}{$r 9.1$} & YV.r & Y 9.7 & r... & 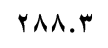 & หัษ.V & rav. 9 & $\mu \ldots$ & كوالح \\
\hline & rA.r & r.r & $r \varepsilon . \varepsilon$ & & YVY.Y & $r \cdot \Lambda .0$ & M 19.0 & المتوسط \\
\hline \multicolumn{2}{|c|}{ 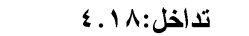 } & ماء & مخلفات: & \multicolumn{4}{|c|}{ 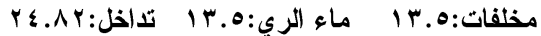 } & LSD .05 \\
\hline
\end{tabular}

$$
\text { ـ." متوسط وزن ... }
$$

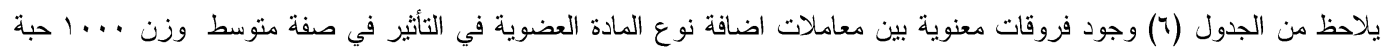

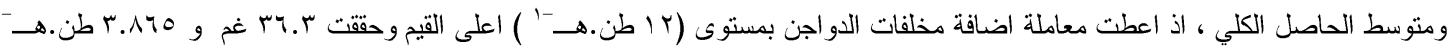

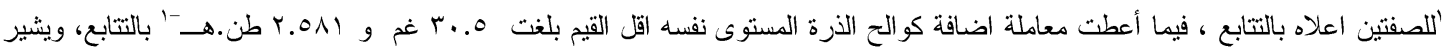

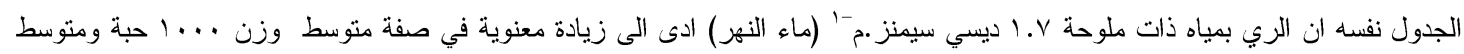

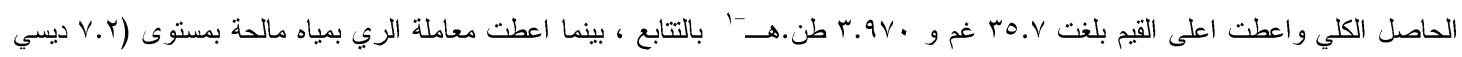

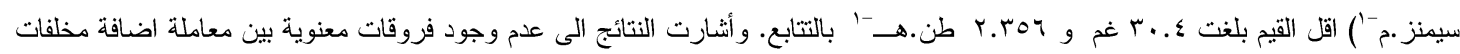

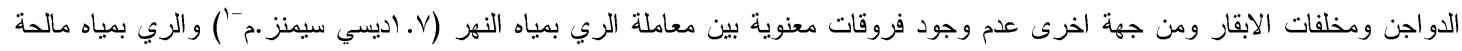

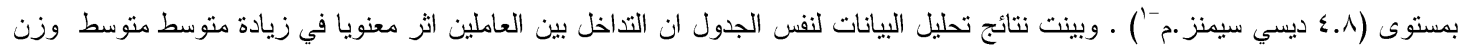

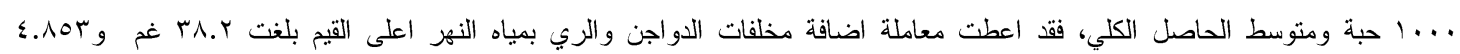

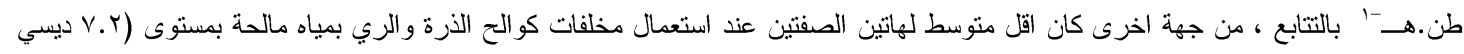

جدول (†) تأثير نوع المخلفات وملوحة ماء الري وتلاخلهما في متوسط وزن ... 1 حبة والحاصل الكلي

\begin{tabular}{|c|c|c|c|c|c|c|c|c|}
\hline \multicolumn{4}{|c|}{ الحاصل الكلي طن.هـ-' } & \multicolumn{4}{|c|}{ وزن ... } & \multirow{3}{*}{ المخلفات } \\
\hline \multicolumn{4}{|c|}{ dS.mم ملوحة ماء الري } & \multicolumn{4}{|c|}{ dS.m-1 ملوحة ماء الري } & \\
\hline المتوسط & V.r & $\varepsilon . r$ & $1 . \mathrm{r} \Lambda$ & المتوسط & V.r & $\varepsilon . r$ & $1 . r \wedge$ & \\
\hline r.Аร० & r.Vos & r.qAV & 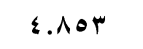 & r. & rr.l & rv.o & rA.r & دواجن \\
\hline r.ru. & Y.rTV & r.ovo & $\varepsilon \ldots \wedge \wedge$ & $r r . \Lambda$ & $r \cdot . \Lambda$ & $r \varepsilon . r$ & r.. & ابقار \\
\hline \multirow[t]{2}{*}{ Y.०人1 } & 1.911 & 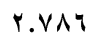 & Y.941 & $r \cdot .0$ & YV.\& & $\mathrm{r} 1.7$ & rY.o & كوالح \\
\hline & Y.rot & r. $\{0$. & r.9V. & & $r \cdot . \varepsilon$ & $r \varepsilon . \varepsilon$ & ro.v & المتوسط \\
\hline \multicolumn{2}{|c|}{ تاخل:r +. } & ماء الز & مخلفات:r 7. & \multicolumn{2}{|c|}{ تذاخل:9 . .๑ } & 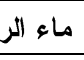 & مخلفات 9 & LSD .05 \\
\hline
\end{tabular}

r.0. متوسط الحاصل البايولوجي (طن.هـ-1') ودليل الحصاد\%

يلاحظ من الجدول (V) وجود فروقات معنوية بين معاملات اضافة نوع المادة العضوية في التأثير في صفة منوسط الحاصل البايولوجي

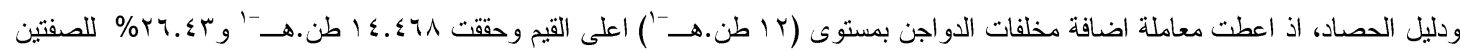

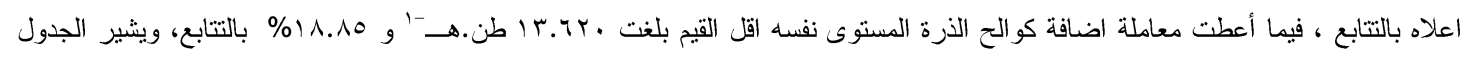

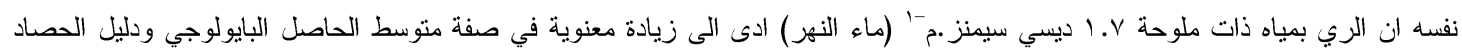

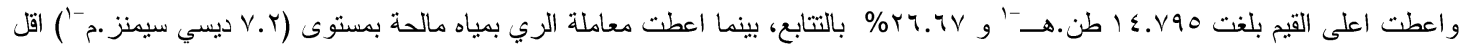

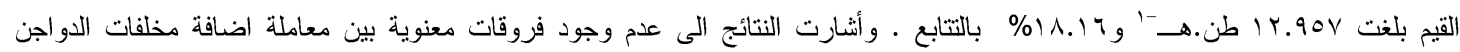




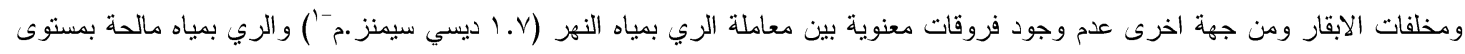

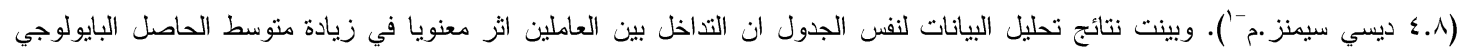

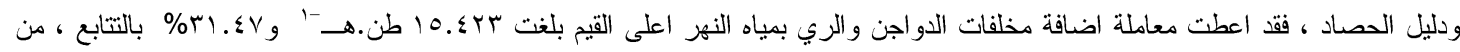

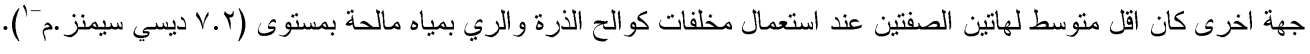

جدول (V) تأثيرنوع المخلفات وملوحة ماء الري وتداخلهما في متوسط الحاصل البيولوجي ودليل الحصاد

\begin{tabular}{|c|c|c|c|c|c|c|c|c|}
\hline \multicolumn{4}{|c|}{ دليل الحصاد % } & \multicolumn{4}{|c|}{ الحاصل البايولوجي طن.هـ-1 } & \multirow{3}{*}{ المخغ أملفات } \\
\hline \multicolumn{4}{|c|}{ dS.m-1 ملوحة ماء الري } & \multicolumn{4}{|c|}{ dS.mم ملوحة ماء الري } & \\
\hline المتوسط & V.r & $\varepsilon . r$ & $1 . r$ & المتوسط & V.r & $\varepsilon . \mu$ & 1.r & \\
\hline$r y . \leq r$ & YI..E & ru.VV & T. & $\mid \varepsilon . \varepsilon 71$ & $11 . .19$ & $1 \varepsilon . \wedge 9 T$ & $10 . \varepsilon r r$ & دواجن \\
\hline צוr.ry & $18 . \wedge 9$ & $r \varepsilon . V T$ & YV. $\leqslant \uparrow$ & 15.110 & $1 r \ldots$ & $1 \leq . \leq 0 \leq$ & $1 \varepsilon . \wedge \wedge 0$ & ابقار \\
\hline \multirow[t]{2}{*}{11.10} & 10.07 & 19.19 & $r 1 . .1$ & ת.ד. & Ir.VVo & $1 \leq \ldots 7$ & $\mid \varepsilon \ldots . \vee \wedge$ & كوالت \\
\hline & 11.17 & (r.^. & rY.TV & & Ir.90V & $|\varepsilon . \leq 0|$ & 15.1990 & المتوسط \\
\hline \multicolumn{2}{|c|}{ تداخل:Y.r } & لاء الري & مخلفات:9 ץ.r & \multicolumn{2}{|c|}{ تداخل:Av. . } & ماء الري: & مخلفات:^^ء . . & LSD .05 \\
\hline
\end{tabular}

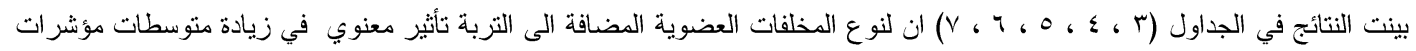

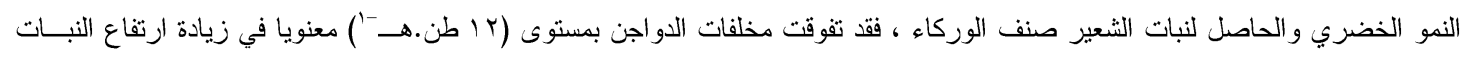

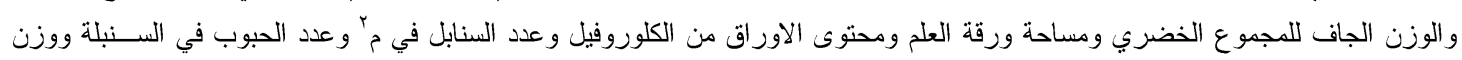

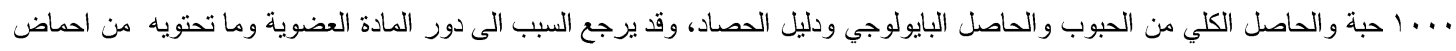

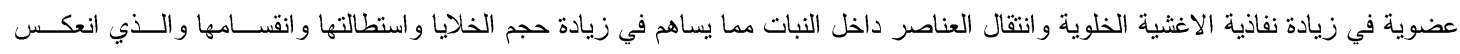

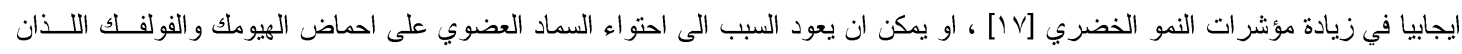

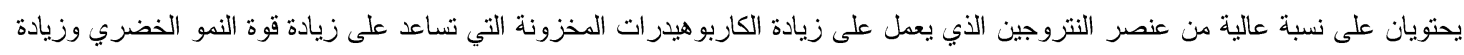

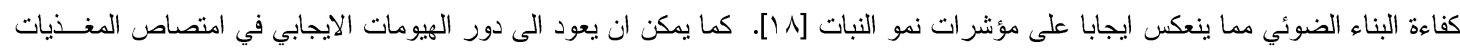

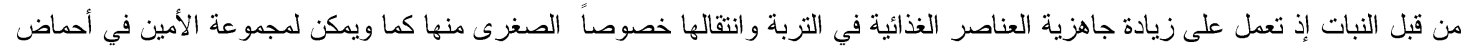

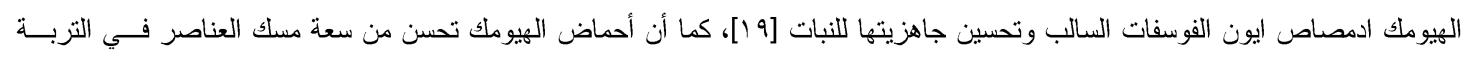

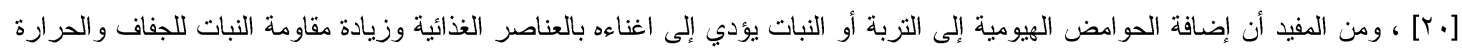

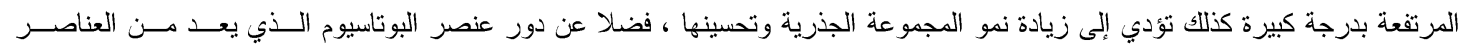

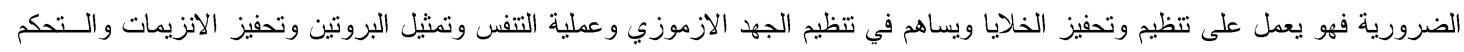

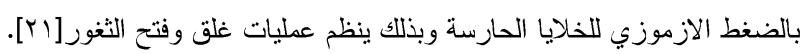

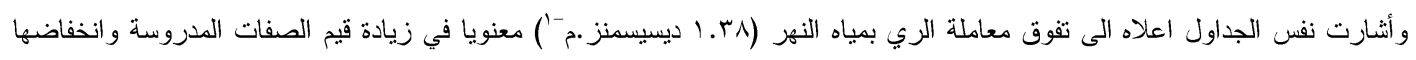

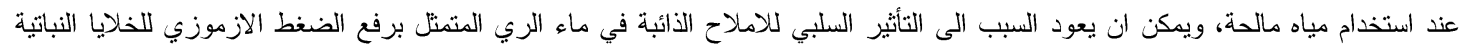

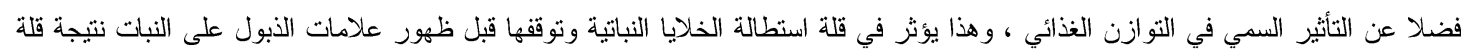

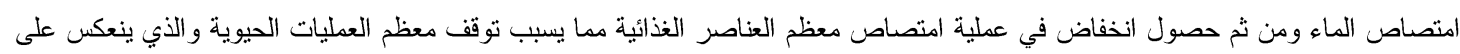

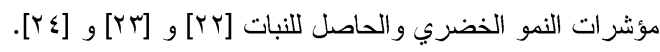

ع - الاستنتاجات

يستتج بان استعمال المخلفات العضوية قد قلل من التأثير السلبي على نمو النبات عند الري بمياه مالحة وان استعمال مخلفات الدواجن

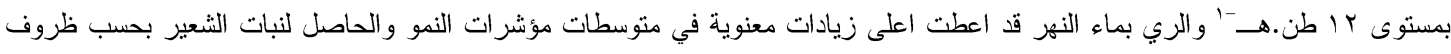

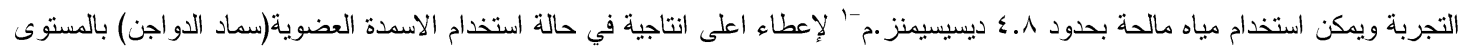
المذكور في الدر اسة. 
[1]الحسن، عباس مهدي و محفوظ عبد القادر محمد. حاصل علف وحبوب نزاكيب وراثية من الثعبر تحت الظروف الديمية. مجلة زراعة

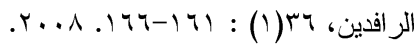

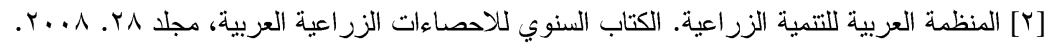

[3]Al-Uqaili , J.K. ; A.K.A. Jarallah ; B.H. Al-Ameri and F.A. Kredi .Effect of saline rainage water on wheat growth and on soil salinity. Iraqi J. Agric. 7(2): 157-166. 2002.

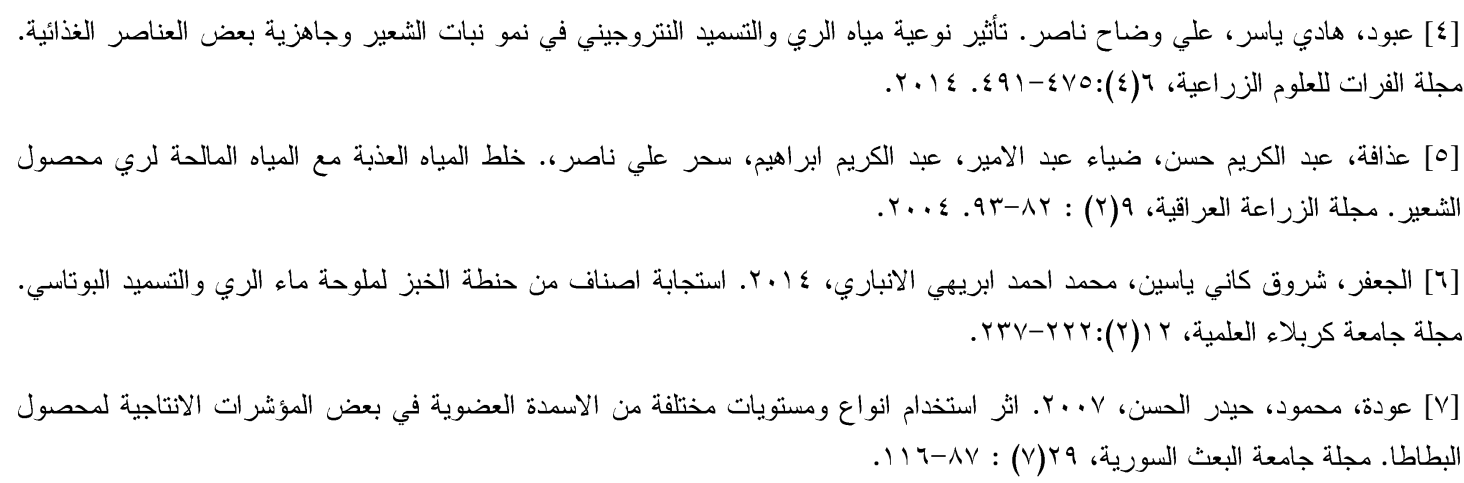

[8]Tejada, M. C. and J. L. Gonzales. Effect of application two organo-mineral fertilizers on nutrient leaching losses and wheat crop., Agron. J. 97 : 960—967. 2005.

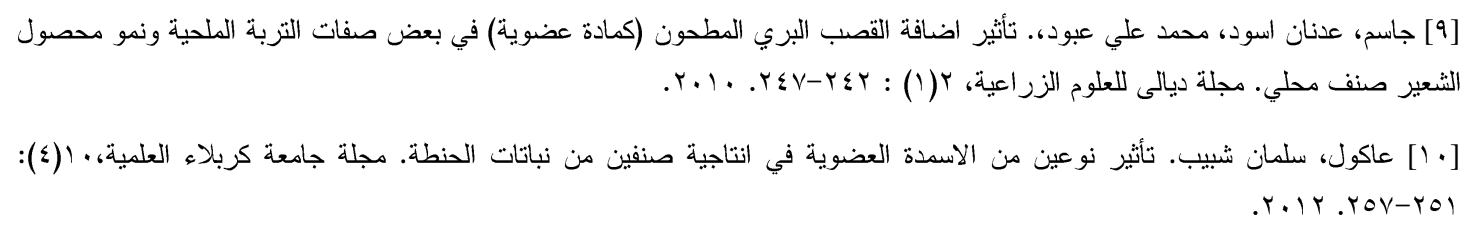

[11] اليونس، عبد الحميد احمد. انتاج وتحسين المحاصيل الحقلية. وزارة التعليم العالي والبحث العلمي، جامعة بغداد، كلية الزراعة، العراق.

[12] Black, C.A.Ed. Methods of Soil Analysis. Part 2.Amer.Soc. Agro. Madison, Wisconson.USA. 1965.

[13] Jackson, M.L. Chemical Analysis. prentice Hall Inc. Englewood cliffs. N.J. 1958.

[14] Thomas, H. The growth response weather of simulated vegetative swards of single genotype of (Lolium perenne). J. Agric., Sci. 84: 333 - 343. 1975.

[10] الر اوي، خاثع محمود و عبد العزيز محمد خلف الله. تصميم وتحليل التجارب الزراعية. كلية الزراعة و الغابات. جامعة الموصل. العراق.

[16] S.A.S. SAS , Users Guide for Personal Computers. Release 7.0. SAS Institute Inc., Cary, NC., USA. (SAS = Statistical Analysis System). 2004.

[17]Wample, R.L., S.E. Spayed, R.G.Evans , and R.G. Steevenc . Nitrogen fertilization and factor influencing grape vine cold hardiness. Inter. Symposium on nitrogen in grapes and wine.Vitic, Davis, USA. 1991.

[18] Keller, M.and M. Kolet. Dry matter and leaf area partitioning bud fertility and Second season growth Vitis vinifera L. : Response to nitrogen supply and limiting irradiance.Vitis. 34 (2) : 77-83. 1995.

[19]Tatini, M.; P. Bertoni; A. Landi and M. L. Traversi. Effect of humic acid on growth and biomass portioning of container-grown olive plants. Acta Hort . 294: 75-80. 1991.

[20]Nardi, S. ; D. Pizzeghello, ; A. Muscolo, and A. Vianello . Physiological effects of humic substances in plant growth. Soil Biol. Biochem. Exeter 34: 1527-1537. 2002.

[21]Barakat, M.R. ; Yehia, T.A. and Sayed, B.M. Response of Newhall Naval Orange to Bio- rganic Fertilization under Newly Reclaimed Area Conditions I:Vegetative Growth and Nutritional Status. Journal of Horticultural Science \&Ornamental Plants, 4 (1): 18-25. 2012. 
[بr] المعموري، عبد الباقي داود سلمان،. تأثثر السماد الفوسفاتي ونسجة التربة ومصدر ماء الري في بعض صفات التربة الكيميائية والخصوبية

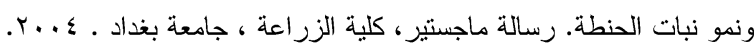

[بr] شكري، حسين محمود،. تأثير استخدام المياه المالحة بالتتاوب والخلط في نمو نبات الحنطة وتر اكم ى الاملاح في التزبة. اطروحة دكتوراه،

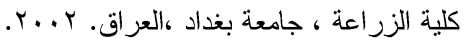

[24] Davis, M. O. and E. T. Nilsen. The physiology of plant under stress. John Wiley and sons , USA. 2000. 